\title{
Hemorrhagic Complications in Pediatric Cardiac Patients on Extracorporeal Membrane Oxygenation: An Analysis of the Extracorporeal Life Support Organization Registry
}

\author{
David K. Werho, MD ${ }^{1}$, Sara K. Pasquali, MD, MHS ${ }^{1}$, Sunkyung Yu, MS ${ }^{1}$, Janet Donohue, \\ MPH $^{1}$, Gail M. Annich, MD ${ }^{2}$, Ravi R. Thiagarajan, MBBS, MPH $^{3}$, Jennifer C. Hirsch-Romano, \\ MD, MS ${ }^{1}$, and Michael G. Gaies, MD, MPH ${ }^{1}$ on behalf of the Extracorporeal Life Support \\ Organization Member Centers \\ ${ }^{1}$ University of Michigan, Ann Arbor, Ml \\ ${ }^{2}$ Toronto Hospital for Sick Children, Toronto, Canada \\ ${ }^{3}$ Boston Children's Hospital, Boston, MA
}

\begin{abstract}
Objectives-To determine the prevalence of and risk factors for hemorrhagic complications in children with cardiac disease requiring extracorporeal membrane oxygenation.
\end{abstract}

Design-Retrospective review of the Extracorporeal Life Support Organization Registry (20022013).

\begin{abstract}
Setting-Participating Extracorporeal Life Support Organization centers.
Patients-Patients less than 18 years old on extracorporeal membrane oxygenation.

Interventions-None.
\end{abstract}

Measurements and Main Results-Of 21,845 patients requiring extracorporeal membrane oxygenation during the study period, 8,905 (41\%) had cardiac disease, and 79\% of whom $(6,995)$ had cardiac surgery. Hemorrhagic complications occurred in 8,480 patients (39\% of overall cohort), with higher rates in cardiac versus noncardiac patients ( $49 \%$ vs $32 \% ; p<0.0001)$ related to cannulation and surgical site bleeding. Cardiac surgical patients had higher rates of hemorrhage compared with cardiac medical patients ( $57 \%$ vs $38 \% ; p<0.0001)$, and cardiac patients with hemorrhage had higher extracorporeal membrane oxygenation mortality compared with those without ( $42 \%$ vs $22 \%$ in medical patients and $34 \%$ vs $20 \%$ in surgical patients; both $p<0.0001$ ). In multivariable analysis in both the cardiac medical and surgical groups, hemorrhage risk was higher in children greater than 1 year old and in patients with longer extracorporeal membrane oxygenation duration. Additional independent risk factors for hemorrhage in cardiac surgical patients included pre-extracorporeal membrane oxygenation mediastinal exploration (odds ratio, 3.6; 95\% CI, 2.1-6.3), Society of Thoracic Surgeons morbidity category 4-5 (odds ratio, 1.2; 95\% CI, 1.03-1.5), cannulation less than 24 hours after surgery (odds ratio, 1.6; 95\% CI, 1.3-1.9), and

For information regarding this article, dwerho@med.umich.edu.

The remaining authors have disclosed that they do not have any potential conflicts of interest. 
longer cardiopulmonary bypass time ( $\geq 282 \mathrm{~min}$ [upper quartile]; odds ratio, 1.5 ; 95\% CI, $1.3-$ $1.9)$.

Conclusions-In this large, multicenter analysis, hemorrhagic complications occurred in nearly half of children with heart disease on extracorporeal membrane oxygenation and were associated with a significant mortality risk. Several factors were associated with hemorrhagic complications in cardiac surgical patients including pre-extracorporeal membrane oxygenation mediastinal exploration, greater surgical complexity, early postoperative cannulation, and longer bypass times. Whether these risks can be mitigated by modifying or delaying systemic anticoagulation requires further investigation.

\section{Keywords}

extracorporeal membrane oxygenation; heart defects; heart diseases; hemorrhage; risk factors

Extracorporeal membrane oxygenation (ECMO) is increasingly used to support critically ill children. Although extracorporeal support can be life saving, children on ECMO are at risk of significant complications which can limit the use of this therapy. Patients on ECMO require systemic anticoagulation to prevent the coagulopathic process initiated when blood contacts an artificial surface, but this therapy predisposes patients on ECMO to lifethreatening hemorrhage (1-3). The frequency of children with complex congenital heart disease or cardiomyopathies requiring ECMO has increased steadily over the past decade (4, 5), and those placed on ECMO after cardiac surgery already have disturbed coagulation pathways and additional surgical sites, factors that have been hypothesized to confer a greater propensity toward bleeding than other ECMO populations (6). Severe bleeding can make ongoing support impossible and may contribute directly to perioperative mortality (7). Therefore, a deeper understanding of the epidemiology and risk factors leading to hemorrhage in pediatric patients with cardiac disease on ECMO is crucial to improving care in this growing population.

Hemorrhage is one of the most common complications of extracorporeal support with a prevalence of $12-52 \%$ in patients on ECMO $(6,8-11)$. CNS hemorrhage particularly increases the risk of in-hospital mortality in these patients (5). Despite the frequency and associated morbidity from these complications in children requiring ECMO, little is known about the prevalence of hemorrhage across diagnostic categories, age groups, and within specific subgroups (notably patients with primary cardiac disease) $(5,10)$. The University of Michigan program previously performed a single-center study analyzing the risk factors for bleeding in postcardiotomy pediatric patients on ECMO (9) and described some early postoperative bleeding risks including longer cardiopulmonary bypass (CPB) times and lower platelet counts. However, many questions remain regarding the impact of patient, disease, and treatment factors on the prevalence of bleeding complications in patients with cardiac disease, particularly, in cardiac surgical patients, and no multicenter investigation of this population has been performed to date. These data are necessary to inform future research and establish evidence-based guidelines for therapy.

To address these knowledge gaps, we performed this study to define the prevalence of hemorrhagic complications in pediatric cardiac patients undergoing ECMO across different 
age groups and diagnostic categories using a multi-institutional database. Under data sponsorship by the Extracorporeal Life Support Organization (ELSO), the ELSO registry data were used to investigate these bleeding rates and to ascertain patient and procedural risk factors for hemorrhage. Our hypothesis was that we could identify high-risk patient populations who might be studied in subsequent prospective investigations aimed at reducing hemorrhagic complications and improving ECMO outcomes. Additionally, we attempted to detect modifiable factors where alternative treatment strategies might reduce the risk of hemorrhage in pediatric cardiac patients.

\section{MATERIALS AND METHODS}

\section{Data Source}

Data were obtained from the ELSO registry under ELSO data sponsorship. The ELSO registry collects data from over 230 international ECMO centers and currently contains information regarding the ECMO runs, patient characteristics, complications, and clinical outcomes for over 50,000 ECMO patients. Data are reported to the ELSO registry by each ECMO center using standardized data collection forms on all patients undergoing ECMO at the participating institutions. For patients undergoing ECMO for a cardiac indication, additional data regarding the patient's cardiac lesions, physiology, surgical course (if applicable), and additional outcomes are submitted via a cardiac addendum. After review by the University of Michigan Hospital and Health Systems institutional review board (IRB), our study was determined to be exempt from IRB regulation as the data were not linked to individual patients and thus was deemed not to be human subject research. For the purposes of this study, deidentified data from all children (age $<18 \mathrm{yr}$ old at the time of ECMO cannulation) reported to the registry between January 2002 and April 2013 were included. Data from subsequent ECMO runs after an initial run in the same patient and data from patients with missing or data entry errors with respect to ECMO duration were excluded ( $n=$ $582)$.

\section{Study Population}

Defining Cardiac Patients-Based on a detailed review of diagnoses in the database, in an effort to ensure capture of patients with primary cardiac disease regardless of indication for ECMO, the cardiac cohort was selected based on the presence of a combination of International Classification of Diseases, 9th Revision (ICD-9) diagnostic codes and Current Procedural Terminology (CPT) procedure codes captured in the database. We identified patients as having primary cardiac disease if they met any of the following criteria:

1. A primary diagnosis ICD-9 code indicating myocarditis, cardiomyopathy, dysrhythmias (excluding only cardiac arrest with no other cardiac codes), congenital cardiac anomalies or cardiac valvar abnormalities (excluding only patent ductus arteriosus [PDA] with no other cardiac codes), or heart transplant recipients.

2. A CPT code indicating cardiac surgery, except patients whose only cardiac CPT code included PDA closure, cardiac procedure not otherwise specified, pericardiocentesis, extracorporeal support, drainage of heart sac, or incision of heart sac. 
3. A CPT code indicating a cardiac catheterization procedure.

4. A secondary diagnosis ICD-9 code indicating myocarditis, cardiomyopathy, or heart transplant recipient.

We assigned all patients with any code for congenital diaphragmatic hernia or repair of congenital diaphragmatic hernia to the noncardiac group. Patients with a secondary diagnosis of dysrhythmias, congenital cardiac abnormalities, or cardiac valvar anomalies were also considered noncardiac unless they had a cardiac procedure code, as the majority of these patients had other significant diseases driving the need for ECMO unrelated to their cardiac disease. For example, we did not include premature infants with hemodynamically insignificant cardiac lesions such as isolated unrepaired atrial septal defects in the cardiac group.

Defining Cardiac Medical and Surgical Patients-Within the cardiac cohort, the patients were divided into medical and surgical groups based on the following criteria:

1. Surgical patients were those with any ECMO run less than 6 months following a surgical procedure that occurred during the same hospitalization. This included those who had surgery after cannulation for ECMO who continued to be supported on ECMO postoperatively, defined by a cardiac surgical CPT code, or patients with an ELSO cardiac addendum procedure code noting any cardiac surgery within the designated timeframe.

2. Patients who had a cardiac surgical procedure code indicating that their surgical procedure occurred at the conclusion of their ECMO run and patients with no surgical procedure codes were considered cardiac medical patients, as any surgical risk conferred on this group would have occurred after the conclusion of their ECMO run.

\section{Primary and Secondary Outcomes}

Our primary outcome was a composite outcome of any hemorrhagic complication on ECMO. These complications are reported by each submitting center and included hemorrhagic death (defined as death after withdrawal of ECMO support due to intractable bleeding), organ site hemorrhages (gastrointestinal [GI] hemorrhage, cannulation site bleeding, pulmonary hemorrhage, CNS hemorrhage [by ultrasound or CT]), and surgical site hemorrhages (surgical site bleeding by report, presence of any procedural CPT code for mediastinal re-exploration for bleeding, or hemorrhagic tamponade by report). To evaluate the most clinically significant complications, we combined CNS hemorrhage and hemorrhagic death into a secondary outcome variable. Mortality data were collected from the registry, and ECMO mortality was defined as death occurring on ECMO or withdrawal of ECMO in anticipation of death, whereas ECMO recovery was defined as successful decannulation without immediate death or anticipation of death.

\section{Defining Variables for Analysis}

Patients within the cardiac cohort were categorized as structural heart disease patients based on the presence of any ICD-9 code for structural heart disease. Patients were also grouped 
by age: neonates ( $0-28 \mathrm{~d}$ old), infants ( $29 \mathrm{~d}$ to $12 \mathrm{mo}$ old), or children ( $>1$ to $<18 \mathrm{yr}$ old). All surgical patients were classified into Society of Thoracic Surgeons (STS) morbidity categories (12) based on their highest risk procedure performed prior to or on ECMO. The STS morbidity categories empirically group operations by associated morbidity risk (category $1=$ lowest risk and category $5=$ highest risk). Baseline demographics, patient characteristics, pre-ECMO and ECMO characteristics and outcomes, and procedural characteristics (for surgical patients), were extracted from the ELSO database. Surgical procedures in which an incision was made on the aorta (beyond simple aortic cannulation for bypass) were considered aortic surgeries for the purposes of analysis.

\section{Statistical Analysis}

Prevalences of hemorrhage were reported as the cumulative prevalence proportion and compared across patient groups using chi-square test. After performing a sensitivity analysis, we excluded patients whose indication for cannulation included pulmonary support or extracorporeal cardiopulmonary resuscitation (ECPR) $(n=4,192)$ beyond the initial descriptive analysis for prevalence of complications; we could not include them in the multivariable analysis for potential surgical risk factors because of high rates of missing data on key predictor variables. Within the cardiac cohort, univariate comparisons of patient, clinical, and procedural characteristics (in the cardiac surgical patients) were made between patients with and without any hemorrhagic complications in the medical patients and surgical patients separately, using chi-square test or Fisher exact test for categorical variables and Wilcoxon rank sum test or $t$ test for continuous variables. Continuous variables including ECMO duration, time from surgery to ECMO cannulation, and CPB time were also evaluated categorically using quartiles.

Variables found to be significantly associated with any hemorrhagic complications in univariate analyses $(p<0.15)$ were subsequently included in multivariable logistic regression to determine independent associations of risk factors with any hemorrhagic complications. Variables with greater than $10 \%$ missing data or those with a zero frequency were not included in the multivariable model. Multicollinearity for the variables included in the multivariable model was checked prior to performing the analysis using variance inflation factor (VIF), which is an indication of multicollinearity if VIF greater than 10, and VIF for each variable in the model was less than 1.5 , which was acceptable to include all variables in the model. Similar analysis was performed to identify independent risk factors for hemorrhagic death and/or CNS hemorrhage in cardiac surgical patient and cardiac medical patients. All analyses were performed using SAS version 9.3 (SAS Institute, Cary, $\mathrm{NC}$ ), with statistical significance set at $p$ values less than 0.05 using two-sided tests.

\section{RESULTS}

A total of 21,845 patients were included in the initial analysis, of whom 8,905 (41\%) had cardiac disease. Patient, pre-ECMO, ECMO, and procedural (where applicable) characteristics are presented in Tables 1 and 2. Of the cardiac patients, 4,170 (47\%) were neonates, 7,277 (82\%) had structural heart disease, and 6,995 (79\%) had cardiac surgery during their ECMO admission. The majority of cardiac patients had venoarterial (VA) 
ECMO (97\%), and their ECMO duration varied considerably (median, $111 \mathrm{hr}$; interquartile range, $63-184 \mathrm{hr}$ ). The ECMO mortality rates were $25 \%$ for the total cohort and $28 \%$ for the cardiac cohort.

\section{Hemorrhage Prevalence in Cardiac Versus Noncardiac Patients}

Overall, $39 \%$ of patients had a hemorrhagic complication, with a higher prevalence in cardiac patients compared with that in noncardiac patients ( $49 \%$ vs $32 \% ; p<0.0001)$. This difference was attributable to higher prevalence of surgical site $(32 \%$ vs $9 \% ; p<0.0001)$ and cannulation site $(17 \%$ vs $14 \% ; p<0.0001)$ bleeding in cardiac patients. Aside from surgical site and cannulation site bleeding, the most common organ system hemorrhage was CNS hemorrhage, and the prevalence did not differ between groups (cardiac $9.3 \%$ vs noncardiac $9.7 \% ; p=0.4$ ). GI hemorrhage and hemorrhagic death occurred more commonly in noncardiac patients (Fig. 1A).

\section{Hemorrhage Prevalence Among Cardiac Subgroups}

Hemorrhage prevalence within the cardiac group was higher in patients with structural heart disease compared with those without structural disease (50\% vs $40 \% ; p<0.0001)$, again related to increased surgical site hemorrhage in structural heart disease patients (36\% vs $14 \% ; p<0.0001)$. However, the prevalence of CNS bleeding was also significantly higher in the structural heart disease group $(10 \%$ vs $6 \% ; p<0.0001)$ (Fig. $1 B)$. The prevalence of all hemorrhagic complications among different age groups was similar (neonates $48 \%$ vs infants $48 \%$ vs children $50 \%$; $p=0.15$ ); however, neonates were more likely to experience hemorrhagic death ( $3.4 \%$ vs $1.9 \% ; p<0.0001)$, CNS hemorrhage ( $13 \%$ vs $5.6 \% ; p<$ 0.0001 ), and surgical site hemorrhage ( $34 \%$ vs $29 \% ; p<0.0001$ ) compared with children greater than 1 year old (Fig. 1C).

Prior to subsequent analysis, sensitivity analysis was performed to compare baseline characteristics between patients included in the cardiac surgical cohort (after excluding ECPR and pulmonary patients) and the overall cardiac cohort, and there were no significant differences except with respect to presence of known genetic anomalies (5.6\% in analyzed patients vs $4.8 \%$ in all cardiac patients; $p=0.048$ ). Figure $1 D$ shows the prevalence of hemorrhagic complications in cardiac medical and cardiac surgical subgroups after the exclusion of ECPR and pulmonary ECMO patients. The surgical group had a higher overall hemorrhage prevalence (57\% vs 38\%; $p<0.0001$ ), again related to increased surgical site hemorrhage. The surgical patients also had a higher prevalence of CNS hemorrhage (10\% vs $8 \% ; p=0.02)$; however, the remainder of hemorrhagic complications including hemorrhagic death, GI hemorrhage, cannulation site bleeding, and pulmonary hemorrhage were not significantly different between the two groups. The ECMO mortality rate for cardiac medical patients with any hemorrhagic complication on ECMO was $42 \%$ versus $22 \%$ in those without a hemorrhagic complication $(p<0.0001)$. Similarly, cardiac surgical patients with hemorrhagic complications had an ECMO mortality rate of $34 \%$ versus $20 \%$ in those who did not have a hemorrhage $(p<0.0001)$. 


\section{Risk Factors in Cardiac Medical Patients}

Table 3 shows the results of both univariate and multivariate analyses in cardiac medical patients. In multivariable analysis, the following factors were independently associated with increased risk of having any hemorrhagic complication on ECMO: age greater than 1 year old, longer ECMO duration, and chest cannulation. The only variable independently associated with our combined secondary outcome of CNS hemorrhage and/or hemorrhagic death among the cardiac medical group was neck cannulation (odds ratio, 2.1; 95\% CI, 1.23.7) (Table 4).

\section{Risk Factors in Cardiac Surgical Patients}

Table 5 shows the univariate and multivariate analyses of risk factors for hemorrhage among cardiac surgical patients. In multivariable analysis, the following factors were independently associated with an increased risk of any hemorrhagic complication on ECMO in cardiac surgical patients: age greater than 1 year old, pre-ECMO mediastinal exploration, longer ECMO duration, STS morbidity category 4-5, cannulation within 24 hours after surgery (including patients cannulated in the operating room), and longer CPB time. Characteristics associated with an increased risk of CNS hemorrhage and/ or hemorrhagic death included neonates, no known genetic anomaly, longer ECMO duration, pre-ECMO pulmonary vasodilatory support, and pre-ECMO bicarbonate administration (Table 6).

\section{DISCUSSION}

In this multicenter study, we describe the prevalence of hemorrhagic complications in pediatric ECMO patients and evaluate risk factors associated with hemorrhage across a large cohort of cardiac patients. We found that patients with any hemorrhage have a higher mortality risk than those who do not, highlighting the importance of this complication. The analysis demonstrates that pediatric cardiac patients, especially cardiac surgical patients, have an increased risk of hemorrhagic complications on ECMO compared with noncardiac and cardiac medical patients, in large part due to surgical site or cannulation site bleeding. Cardiac surgical patients are also more likely to have CNS hemorrhage compared with cardiac medical patients.

We identified several risk factors associated with increased hemorrhagic risk, such as age, pre-ECMO illness severity, and surgical risk. Although these factors may not be modifiable, they are useful for identifying high-risk patient subgroups that may be targeted for study in future prospective studies or interventional trials. We did find other potentially modifiable variables impacting the risk of hemorrhage such as cannulation site and duration of ECMO although these are also largely dictated by patient factors. One notable finding was the increased risk of hemorrhage in cardiac surgical patients with longer CPB times, pre-ECMO mediastinal exploration, and those who were cannulated to ECMO within 24 hours of their surgical repair. These data suggest that practice changes in patients with these risk factors may be beneficial; one could consider delayed heparin initiation or modified anticoagulation strategies in these patients soon after cannulation in order to decrease the risk of a hemorrhagic complication, balancing these choices against the increased risk for thrombotic complications in the absence of anticoagulation. 
Prior studies describing overall ECMO complications or outcomes from the ELSO database have reported hemorrhage rates in specific subsets of patients $(5,10,13)$. The reported prevalence of hemorrhage from these small single institutional studies varies widely from $12 \%$ in cardiomyopathy patients (6) to $52 \%$ in ECMO nonsurvivors (8) and postcardiotomy cardiogenic shock patients (11). In this study, we found an overall hemorrhagic complication prevalence of $39 \%$ in all pediatric patients on ECMO, with similar high rates of hemorrhage in cardiac (49\%) and structural heart disease patients (50\%) to other studies. Our cohort of cardiac patients without structural heart disease had a hemorrhage prevalence of $40 \%$, which is higher than the $12 \%$ in cardiomyopathy patients described by Coskun et al (6); however, their study included ventricular assist device patients in the cardiomyopathy cohort, which may explain their lower hemorrhage rates.

Arguably, the most clinically important hemorrhagic complication, CNS hemorrhage, occurs commonly in pediatric patients, with rates of 3-11\% reported in the literature, and higher rates among premature infants and other neonates (up to $36 \%$ in neonates with gestational age $<35$ weeks) $(5,10,14,15)$. This complication potentially alters the clinical course of these patients in substantive ways, representing a major source of mortality and short-term or long-term morbidity, yet no consensus exists on the best prevention or therapeutic strategies. Studies focusing on CNS hemorrhage and neurological injury in pediatric ECMO patients demonstrate several risk factors for CNS injury including neonatal age, sepsis, lower gestational age, acidosis, excessive fluid resuscitation, hypoxia, ECPR, sepsis, and epinephrine use (14-19). In our study, we could not evaluate many of these characteristics due to absence of these variables and missing data in the registry; however, we did demonstrate neonatal age to be a significant risk factor in cardiac surgical patients on ECMO. Additionally, we found pre-ECMO pulmonary vasodilatory support and bicarbonate administration to be risk factors for our combined secondary outcome of CNS hemorrhage or hemorrhagic death in our cardiac surgical cohort. These factors may be surrogate markers for patients with greater physiologic derangement, and it is possible that CNS injury occurs during the pre-ECMO phase but manifests after a patient is supported with ECMO. Interestingly, patients with a known genetic anomaly had less risk of CNS hemorrhage or hemorrhagic death in our analysis; however, this may be due to their propensity to succumb to other causes of death related to other extracardiac organ dysfunction, or for their ECMO course to be discontinued before hemorrhagic complications emerge.

In our study, we found neck cannulation to be an independent risk factor for CNS hemorrhage and hemorrhagic death for cardiac medical patients. The choice to perform neck cannulation is due to many factors that may or may not be related to the patient's underlying disease; thus, there may be confounding by indication of the association between cannulation site and hemorrhage. Additionally, we were unable to determine from the dataset what factors would contribute to a nonsurgical patient undergoing chest cannulation for ECMO. Data on the association between neck cannulation and risk of CNS injury in ECMO patients have been mixed in the literature. Some authors postulate that carotid artery ligation in VA ECMO alters cerebral hemodynamics and blood flow and thus predisposing patients to CNS hemorrhage $(18,20,21)$, whereas others implicate lack of sufficient jugular venous drainage as the predominant risk factor. Other studies show decreased neurologic injury with cephalad jugular venous drainage in venovenous ECMO (22) or no difference 
$(19,23)$. We were not able to determine whether carotid ligation or cephalad jugular venous drainage was performed in each case, and we were unable to control for illness severity due to the absence of important risk-adjustment variables in the registry. Further analysis accounting for these factors is necessary to determine whether any particular cannulation approach is protective for CNS injury in instances where a clinician has a clear choice not dictated by the patient's disease.

Although patient factors and other ECMO techniques contribute to the risk of hemorrhage, no therapy is more directly related to this risk than the provision of pharmacologic anticoagulation. However, there is no multi-institutional registry that provides sufficient data to study these practices. Previous literature describes the wide variance in anticoagulation management and hematologic monitoring techniques between hospitals (24). However, to date, there has been sparse evidence to suggest the comparative risks and benefits of these different strategies as they pertain to risk of hemorrhagic complications (2). An ELSO consensus statement and guidelines on anticoagulation management have recently been developed based on expert consensus and review of multiple anticoagulation practices across ELSO centers $(25,26)$. If the ELSO registry begins to collect data on anticoagulation management and monitoring practices in the future, the impact of anticoagulation monitoring strategies and therapies on ECMO outcomes, particularly hemorrhagic complications, could be assessed using methods similar to those applied in this study, and this would be a ripe area for future research.

The primary limitations of this analysis include all those inherent to doing a retrospective database review, most notably that we could not control for unmeasured confounders. We could only analyze those variables and outcomes included in the database, which contains a limited number of variables on specific care processes, and does not include some factors that may significantly impact bleeding risk, such as anticoagulation strategies, laboratory monitoring strategies, blood product administration, and the use of antifibrinolytics. Best practices in these domains remain an area of debate clinically and in the literature and the impact on outcomes must be ascertained to inform evidence-based practice changes in the future. We did not have access to center volume data to analyze the association of center ECMO experience with bleeding complications although this has been associated with ECMO mortality in other studies $(27,28)$. We also could not account for within-center clustering based on our dataset as center-level identifiers are not released with the data. As mentioned previously, patients whose indication for cannulation included pulmonary support or ECPR did not have cardiac addenda completed and thus did not have the necessary procedural data to be included in our analysis of the surgical cohort. These excluded patients differed from our included patients with regard to presence of known genetic anomalies and may limit generalizability of our data to patients placed on ECMO for ECPR or pulmonary support.

\section{CONCLUSIONS}

Hemorrhagic complications, particularly CNS hemorrhage and hemorrhagic death, remain important morbidities for patients on ECMO and may limit the duration and effectiveness of ECMO support in critically ill patients. Our study demonstrated that the growing population 
of pediatric cardiac surgical patients on ECMO are at increased risk of surgical site bleeding and CNS hemorrhage compared with other pediatric ECMO patients and that the occurrence of any hemorrhagic complication more than doubles ECMO mortality rates. We demonstrated some important risk factors for hemorrhage in pediatric cardiac patients on ECMO, which unfortunately are not modifiable and are related to the nature of congenital heart disease and complex surgical management of structural lesions. However, by identifying these higher risk patients, anticoagulation and other ECMO management strategies may be more critically examined on a case-by-case basis to reduce morbidity and mortality in this group. Currently, the degree to which anticoagulation strategies precipitate or contribute to these complications remains unknown and represents a major target for future research and may be more effectively studied in these high-risk patients to improve care and inform practices in all ECMO patients. A better understanding of these factors will likely lead to important practice changes that result in improved short-term and long-term outcomes for patients requiring ECMO support.

\section{Acknowledgments}

Supported, in part, by internal funding from the University of Michigan Congenital Heart Center.

Dr. Werho received support for travel from the University of Michigan (travel funds to present abstract) Dr. Pasquali (principal investigator) receives support from the National Heart, Lung, and Blood Institute (NIHLBI) (grant K08HL103631); and received support for article research from the National Institutes of Health. Her institution received grant support from NHLBI K08 award. Dr. Gaies (principal investigator) receives support from the NIHLBI (grant K08HL116639). Dr. Thiagarajan lectured for the American Society of Pediatric Hematology. His institution received grant support from the NHLBI Pumpkin Trial. Dr. Hirsch-Romano provided expert testimony for the UCLA Children's Hospital.

We thank the Extracorporeal Life Support Organization for providing the data for this study. We thank Peter Rycus, MPH, for helping navigate the database as well as extracting the data. We thank the Michigan Congenital Heart Outcomes Research and Discovery (M-CHORD) program for database and statistical support.

\section{References}

1. Muntean W. Coagulation and anticoagulation in extracorporeal membrane oxygenation. Artif Organs. 1999; 23:979-983. [PubMed: 10564301]

2. Oliver WC. Anticoagulation and coagulation management for ECMO. Semin Cardiothorac Vasc Anesth. 2009; 13:154-175. [PubMed: 19767408]

3. Gaffney AM, Wildhirt SM, Griffin MJ, et al. Extracorporeal life support. BMJ. 2010; 341:982-986.

4. Suzuki Y, Yamauchi S, Daitoku K, et al. Extracorporeal membrane oxygenation circulatory support after congenital cardiac surgery. ASAIO J. 2009; 55:53-57. [PubMed: 19092671]

5. Haines NM, Rycus PT, Zwischenberger JB, et al. Extracorporeal Life Support Registry Report 2008: Neonatal and pediatric cardiac cases. ASAIO J. 2009; 55:111-116. [PubMed: 19092657]

6. Coskun KO, Coskun ST, Popov AF, et al. Extracorporeal life support in pediatric cardiac dysfunction. J Cardiothorac Surg. 2010; 5:112. [PubMed: 21083896]

7. McMullan DM, Emmert JA, Permut LC, et al. Minimizing bleeding associated with mechanical circulatory support following pediatric heart surgery. Eur J Cardiothorac Surg. 2011; 39:392-397. [PubMed: 20801051]

8. Reed RC, Rutledge JC. Laboratory and clinical predictors of thrombosis and hemorrhage in 29 pediatric extracorporeal membrane oxygenation nonsurvivors. Pediatr Dev Pathol. 2010; 13:385392. [PubMed: 20085498]

9. Nardell K, Annich GM, Hirsch JC, et al. Risk factors for bleeding in pediatric post-cardiotomy patients requiring ECLS. Perfusion. 2009; 24:191-197. [PubMed: 19767331] 
10. Zwischenberger JB, Nguyen TT, Upp JR, et al. Complications of neonatal extracorporeal membrane oxygenation. Collective experience from the Extracorporeal Life Support Organization. J Thorac Cardiovasc Surg. 1994; 107:838-849. [PubMed: 8127113]

11. Muehrcke DD, McCarthy PM, Stewart RW, et al. Extracorporeal membrane oxygenation for postcardiotomy cardiogenic shock. Ann Thorac Surg. 1996; 61:684-691. [PubMed: 8572788]

12. Jacobs ML, O'Brien SM, Jacobs JP, et al. An empirically based tool for analyzing morbidity associated with operations for congenital heart disease. J Thorac Cardiovasc Surg. 2013; 145:1046e1-1057.e1. [PubMed: 22835225]

13. Kim K, Mazor RL, Rycus PT, et al. Use of venovenous extracorporeal life support in pediatric patients for cardiac indications: A review of the Extracorporeal Life Support Organization registry. Pediatr Crit Care Med. 2012; 13:285-289. [PubMed: 21926665]

14. Hervey-Jumper SL, Annich GM, Yancon AR, et al. Neurological complications of extracorporeal membrane oxygenation in children. J Neurosurg Pediatr. 2011; 7:338-344. [PubMed: 21456903]

15. Raets MM, Dudink J, Ijsselstijn H, et al. Brain injury associated with neonatal extracorporeal membrane oxygenation in The Netherlands: A nationwide evaluation spanning two decades. Pediatr Crit Care Med. 2013; 14:884-892. [PubMed: 24121484]

16. de Mol AC, Gerrits LC, van Heijst AF, et al. Intravascular volume administration: A contributing risk factor for intracranial hemorrhage during extracorporeal membrane oxygenation? Pediatrics. 2008; 121:e1599-e1603. [PubMed: 18458037]

17. Dela Cruz TV, Stewart DL, Winston SJ, et al. Risk factors for intracranial hemorrhage in the extracorporeal membrane oxygenation patient. J Perinatol. 1997; 17:18-23. [PubMed: 9069059]

18. Barrett CS, Bratton SL, Salvin JW, et al. Neurological injury after extracorporeal membrane oxygenation use to aid pediatric cardiopulmonary resuscitation. Pediatr Crit Care Med. 2009; 10:445-451. [PubMed: 19451851]

19. Hardart GE, Fackler JC. Predictors of intracranial hemorrhage during neonatal extracorporeal membrane oxygenation. J Pediatr. 1999; 134:156-159. [PubMed: 9931522]

20. Mendoza JC, Shearer LL, Cook LN. Lateralization of brain lesions following extracorporeal membrane oxygenation. Pediatrics. 1991; 88:1004-1009. [PubMed: 1945603]

21. Teele SA, Salvin JW, Barrett CS, et al. The association of carotid artery cannulation and neurologic injury in pediatric patients supported with venoarterial extracorporeal membrane oxygenation*. Pediatr Crit Care Med. 2014; 15:355-361. [PubMed: 24622166]

22. O'Connor TA, Haney BM, Grist GE, et al. Decreased incidence of intracranial hemorrhage using cephalic jugular venous drainage during neonatal extracorporeal membrane oxygenation. J Pediatr Surg. 1993; 28:1332-1335. [PubMed: 8263697]

23. Skarsgard ED, Salt DR, Lee SK. Extracorporeal Life Support Organization Registry: Venovenous extracorporeal membrane oxygenation in neonatal respiratory failure: Does routine, cephalad jugular drainage improve outcome? J Pediatr Surg. 2004; 39:672-676. [PubMed: 15136997]

24. Bembea MM, Annich G, Rycus P, et al. Variability in anticoagulation management of patients on extracorporeal membrane oxygenation: An international survey. Pediatr Crit Care Med. 2013; 14:e77-e84. [PubMed: 23287906]

25. Annich G, Adachi I. Anticoagulation for pediatric mechanical circulatory support. Pediatr Crit Care Med. 2013; 14:S37-S42. [PubMed: 23735984]

26. Lequier, L.; Annich, G.; Al-Ibrahim, O., et al. [Accessed October 2, 2014] ELSO Anticoagulation Guidelines. Available at: http://www.wwww.elso.org/resources/guidelines?download=606:elsoanticoagulation-guidelines

27. Freeman CL, Bennett TD, Casper TC, et al. Pediatric and neonatal extracorporeal membrane oxygenation: Does center volume impact mortality?*. Crit Care Med. 2014; 42:512-519. [PubMed: 24164955]

28. Karamlou T, Vafaeezadeh M, Parrish AM, et al. Increased extracorporeal membrane oxygenation center case volume is associated with improved extracorporeal membrane oxygenation survival among pediatric patients. J Thorac Cardiovasc Surg. 2013; 145:470-475. [PubMed: 23246046] 


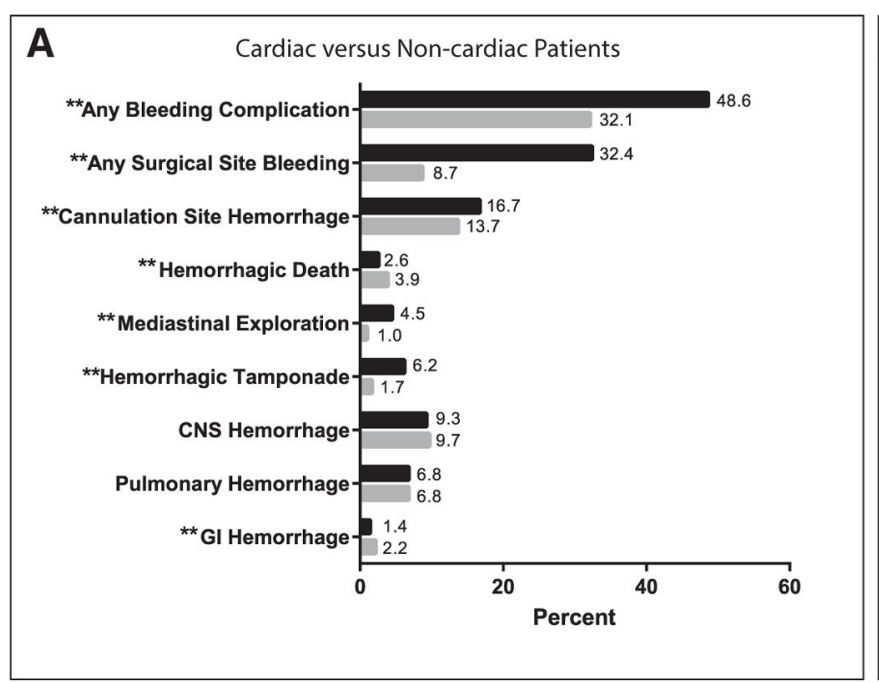

B Structural Heart Disease versus Non-structural Heart Disease Patients
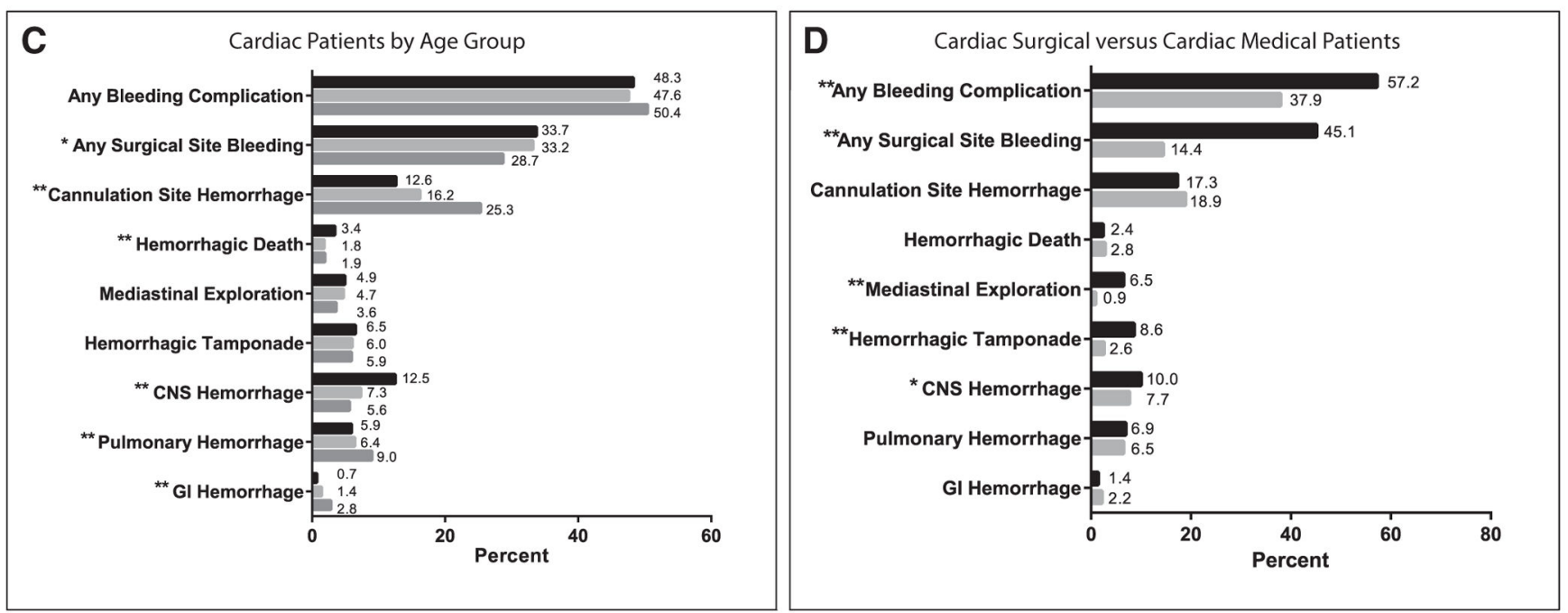

Figure 1.

Prevalence of hemorrhage in different patient groups, categorized by complication type.

Values are expressed as the percentage of patients within each cohort who experienced a hemorrhagic complication during their extracorporeal membrane oxygenation hospitalization $(* p<0.05 ; * *<0.0001)$. A, Cardiac versus noncardiac patients. Dark bars represent cardiac patients $(n=8,905)$; light bars represent noncardiac patients $(n=12,940)$. B, Structural heart disease versus nonstructural cardiac patients. Dark bars represent structural heart disease patients $(n=7,277)$; light bars represent nonstructural heart disease patients $(n=1,406)$. C, Cardiac patients by age group. Dark bars represent neonates 0-28 d old $(n=4,170)$; light bars represent infants $29-365 \mathrm{~d}$ old $(n=2,642)$; medium bars represent children greater than $1 \mathrm{yr}$ old to less than $18 \mathrm{yr}$ old $(n=2,093)$. D, Cardiac surgical versus cardiac medical patients, after exclusion of extracorporeal cardiopulmonary resuscitation and pulmonary patients. Dark bars represent cardiac surgical patients $(n=3,517)$; light bars represent cardiac medical patients $(n=1,196)$. GI $=$ Gastrointestinal. 


\section{TABLE 1}

Patient, Pre-extracorporeal Membrane Oxygenation, and During Extracorporeal Membrane Oxygenation Characteristics (Overall Cohort)

\begin{tabular}{|c|c|c|c|c|}
\hline Characteristics & All $(n=21,845)$ & Cardiac $(n=8,905)$ & $\begin{array}{l}\text { Cardiac Surgical }^{a} \\
\quad(n=3,517)\end{array}$ & $\begin{array}{c}\text { Cardiac Medical }^{a}(n \\
=1,196)\end{array}$ \\
\hline Male sex & $12,141(55.6)$ & $4,935(55.4)$ & $1,976(56.2)$ & $638(53.3)$ \\
\hline \multicolumn{5}{|l|}{ Race } \\
\hline Caucasian & $12,004(55.0)$ & $4,964(55.7)$ & $2,003(57.0)$ & $639(53.4)$ \\
\hline African American & $3,789(17.3)$ & $1,310(14.7)$ & 445 (12.7) & $236(19.7)$ \\
\hline Asian & $1,365(6.2)$ & $658(7.4)$ & $254(7.2)$ & $86(7.2)$ \\
\hline Hispanic & $3,097(14.2)$ & $1,243(14.0)$ & $534(15.2)$ & $153(12.8)$ \\
\hline Other & $1,253(5.7)$ & $563(6.3)$ & $208(5.9)$ & $63(5.3)$ \\
\hline Current weight, $\mathrm{kg}$ & $3.6(3.0-6.6)$ & $3.9(3.0-8.0)$ & $3.6(3.0-5.7)$ & $6.0(3.4-16.2)$ \\
\hline \multicolumn{5}{|l|}{ Age group } \\
\hline Neonate $(0-28 \mathrm{~d}$ & $13,121(60.1)$ & $4,170(46.8)$ & $1,902(54.1)$ & $413(34.5)$ \\
\hline Infant (29-365 d) & $4,107(18.8)$ & $2,642(29.7)$ & $1,032(29.3)$ & $290(24.2)$ \\
\hline Child ( $366 \mathrm{~d}$ to $18 \mathrm{yr}$ ) & $4,617(21.1)$ & $2,093(23.5)$ & $583(16.6)$ & $493(41.2)$ \\
\hline Genetic anomaly & $724(3.3)$ & $429(4.8)$ & $238(6.8)$ & $26(2.2)$ \\
\hline \multicolumn{5}{|l|}{ Pre-ECMO } \\
\hline \multicolumn{5}{|l|}{ Pre-ECMO support } \\
\hline Pressor support & $18,699(85.6)$ & $7,592(85.3)$ & $3,020(85.6)$ & $1,018(85.1)$ \\
\hline Cardiac mechanical support & $184(0.8)$ & $156(1.8)$ & $64(1.8)$ & $16(1.3)$ \\
\hline $\begin{array}{l}\text { Oscillator support or high-frequency } \\
\text { oscillation/high-frequency ventilation }\end{array}$ & $9,628(44.1)$ & $671(7.5)$ & $143(4.1)$ & $107(8.9)$ \\
\hline Pulmonary vasodilatory support & $11,736(53.7)$ & $2,397(26.9)$ & $1,026(29.2)$ & $225(18.8)$ \\
\hline Systemic afterload reduction & $5,311(24.3)$ & $3,010(33.8)$ & $1,415(40.2)$ & $340(28.4)$ \\
\hline Steroids & $843(3.9)$ & $247(2.8)$ & $109(3.1)$ & $27(2.3)$ \\
\hline Bicarbonate & $6,309(28.9)$ & $2,472(27.8)$ & $871(24.8)$ & $270(22.6)$ \\
\hline $\begin{array}{l}\text { Pre-ECMO re-exploration for bleeding/ } \\
\text { mediastinal exploration }\end{array}$ & $337(1.5)$ & $223(2.5)$ & $109(3.1)$ & $9(0.8)$ \\
\hline \multicolumn{5}{|l|}{ On ECMO } \\
\hline Duration of ECMO, hr & $134(76-226)$ & $111(63-184)$ & $104(64-167)$ & $128(69-219)$ \\
\hline \multicolumn{5}{|l|}{ ECMO mode } \\
\hline Venoarterial & $17,294(79.2)$ & $8,630(96.9)$ & 3,467 (98.6) & $1,176(98.3)$ \\
\hline Venovenous & $4,389(20.1)$ & $197(2.2)$ & $19(0.5)$ & $9(0.8)$ \\
\hline Other & $107(0.5)$ & $55(0.6)$ & $24(0.7)$ & $8(0.7)$ \\
\hline \multicolumn{5}{|l|}{ Cannulation site } \\
\hline Chest & $6,247(28.6)$ & $5,452(61.2)$ & $2,924(83.1)$ & $329(27.5)$ \\
\hline Neck & $14,376(65.8)$ & $2,863(32.2)$ & $411(11.7)$ & $733(61.3)$ \\
\hline Groin/other & $584(2.7)$ & 305 (3.4) & $44(1.3)$ & $100(8.4)$ \\
\hline
\end{tabular}

Pediatr Crit Care Med. Author manuscript; available in PMC 2016 March 01. 


\begin{tabular}{|c|c|c|c|c|}
\hline Characteristics & All $(n=21,845)$ & Cardiac $(n=8,905)$ & $\begin{array}{l}\text { Cardiac Surgical } \\
\quad(n=\mathbf{3 , 5 1 7})\end{array}$ & $\begin{array}{c}\text { Cardiac Medical }^{a}(n \\
=1,196)\end{array}$ \\
\hline \multicolumn{5}{|l|}{ Clinical outcomes } \\
\hline Recovery to decannulation & $16,297(74.6)$ & $6,364(71.5)$ & 2,519 (71.6) & $839(70.2)$ \\
\hline Death at decannulation & $5,506(25.2)$ & $2,518(28.3)$ & $987(28.1)$ & $353(29.5)$ \\
\hline $\begin{array}{l}\text { Reason for discontinuation ECMO: } \\
\text { hemorrhage }\end{array}$ & $739(13.4)$ & $231(9.2)$ & $86(8.7)$ & $34(9.6)$ \\
\hline
\end{tabular}

$\mathrm{ECMO}=$ extracorporeal membrane oxygenation.

${ }^{a}$ After exclusion of extracorporeal cardiopulmonary resuscitation and pulmonary ECMO patients.

Data are presented as $\mathrm{n}(\%)$ for categorical variables and median (interquartile range) for continuous variables. 
TABLE 2

Procedural Characteristics of the Cardiac Surgical Cohort

\begin{tabular}{|lc|}
\hline Characteristics & Cardiac Surgical $^{\boldsymbol{a}}(\boldsymbol{n}=\mathbf{3 , 5 1 7})$ \\
\hline Society of Thoracic Surgeons morbidity risk category & \\
1 to 3 & $1,419(40.3)$ \\
4 or 5 & $2,005(57.0)$ \\
\hline Time from surgery to extracorporeal membrane oxygenation cannulation, hr & $13(7-23)$ \\
$\leq 7$ (<25th percentile) & $1,041(29.6)$ \\
$7-13$ (25th to 50th percentile) & $722(20.5)$ \\
$13-23$ (50th to 75th percentile) & $885(25.2)$ \\
$>23$ (> 75th percentile) & $869(24.7)$ \\
\hline Aortic surgery & $1,901(54.1)$ \\
\hline Cardiopulmonary bypass time time, min & $193(132-282)$ \\
$<193$ (< 50th percentile) & $1,587(45.1)$ \\
$193-282$ (50th to 75th percentile) & $796(22.6)$ \\
$\geq 282$ (> 75th percentile) & $798(22.7)$ \\
Unknown & $336(9.6)$ \\
\hline
\end{tabular}

${ }^{a}$ After exclusion of patients on extracorporeal cardiopulmonary resuscitation and pulmonary extracorporeal membrane oxygenation. Data are presented as $n(\%)$ for categorical variables and median (interquartile range) for continuous variables. 


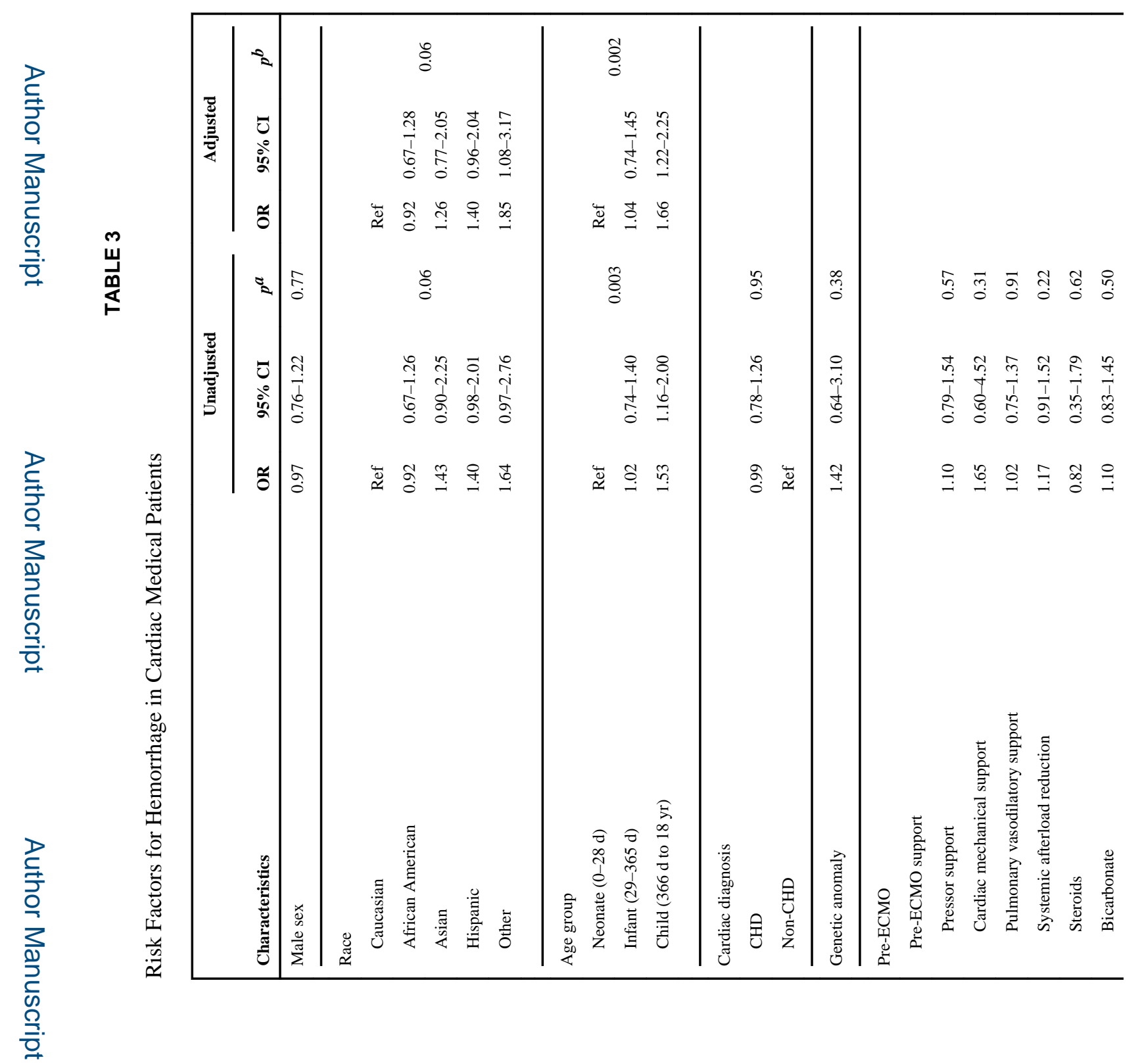

Pediatr Crit Care Med. Author manuscript; available in PMC 2016 March 01. 


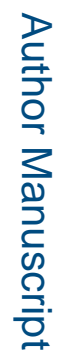

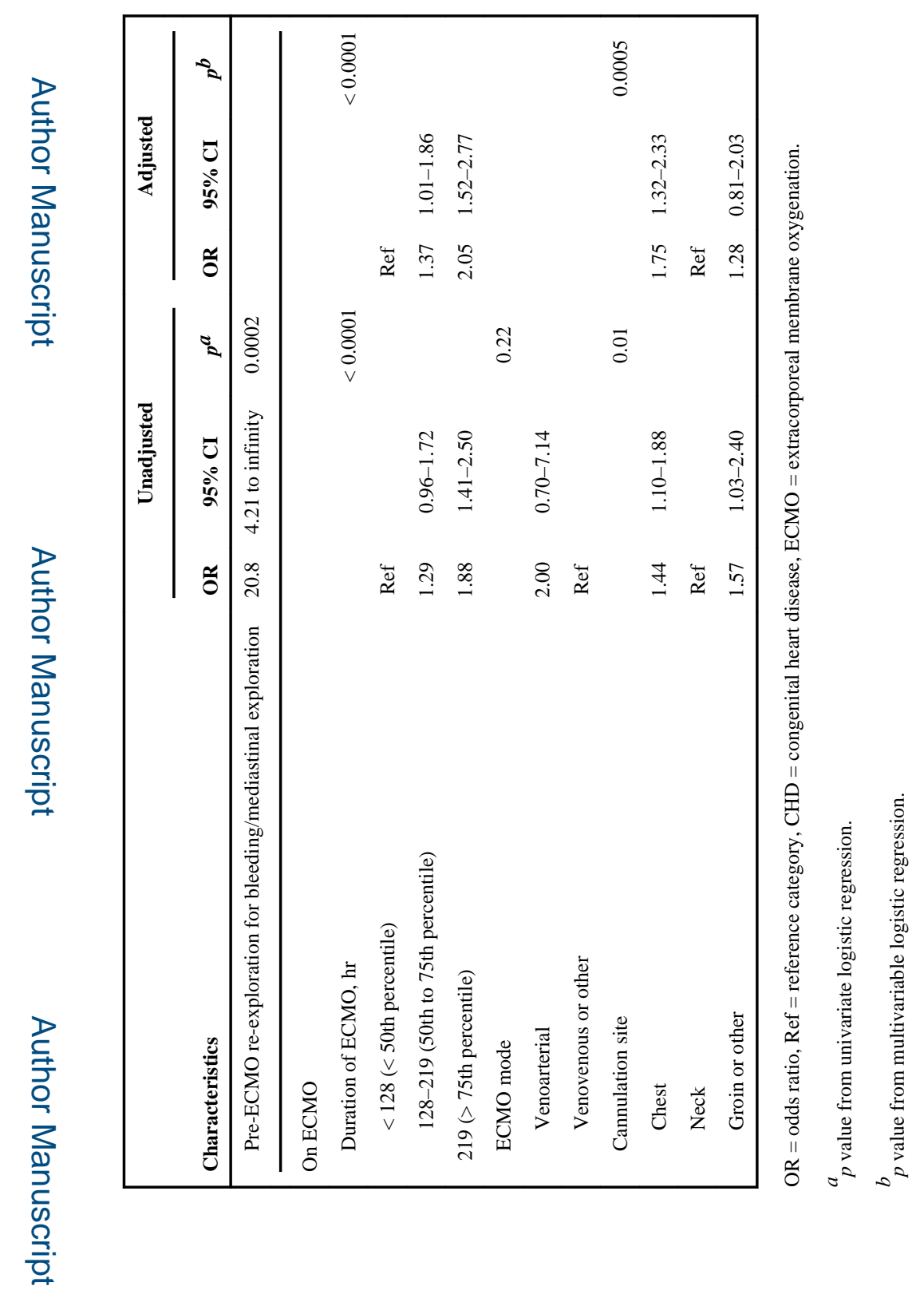

Pediatr Crit Care Med. Author manuscript; available in PMC 2016 March 01. 


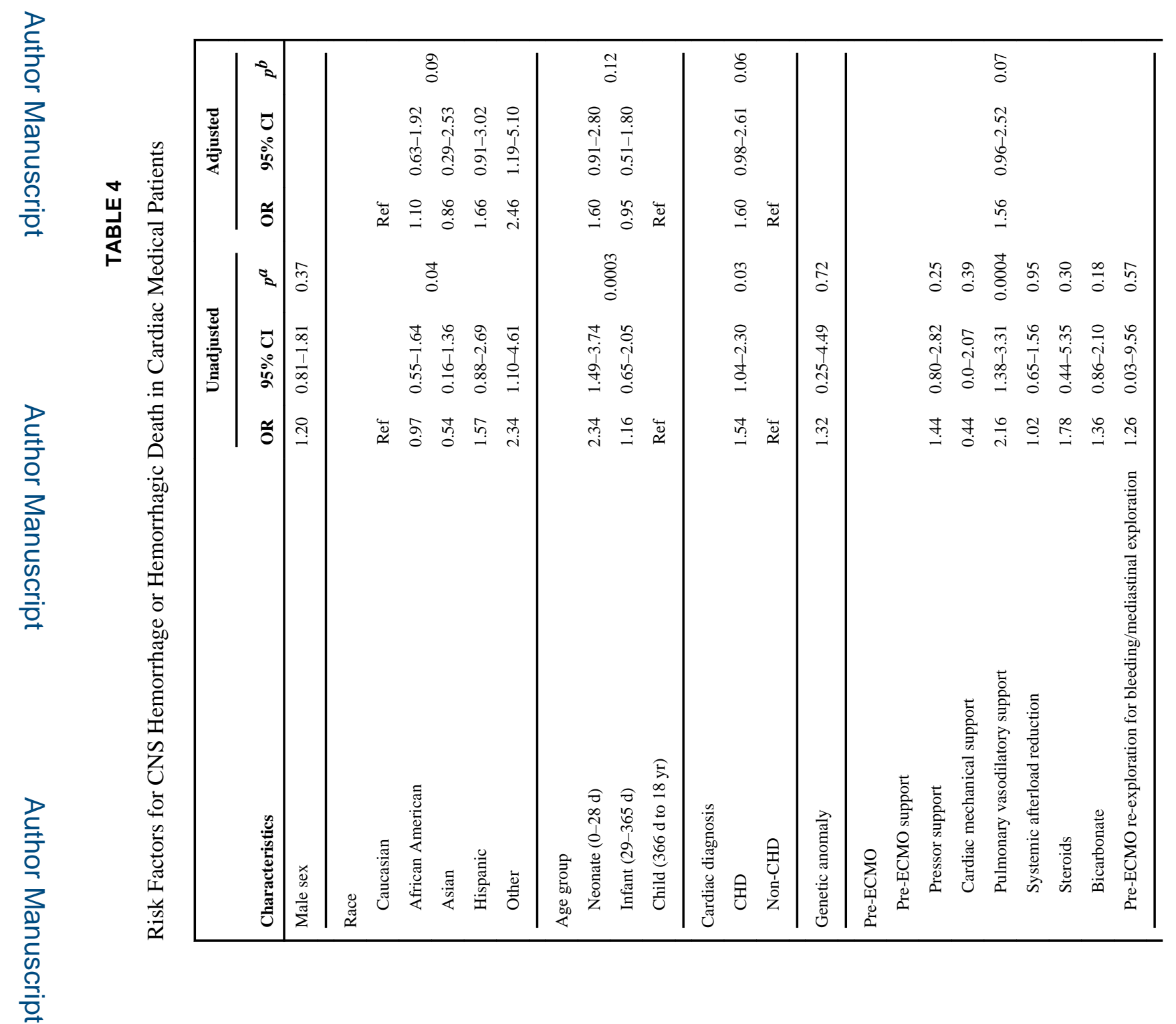

Pediatr Crit Care Med. Author manuscript; available in PMC 2016 March 01. 


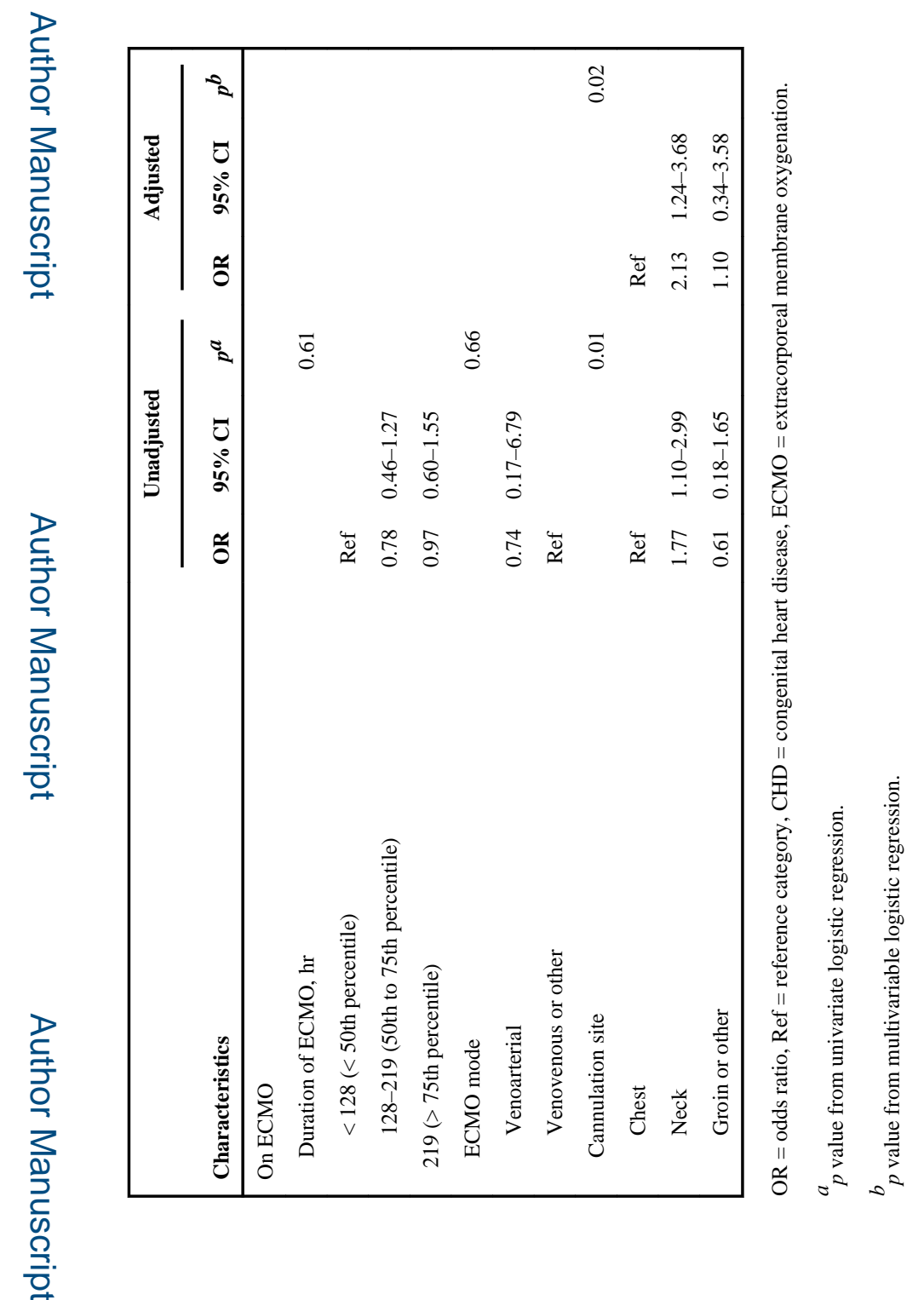

Pediatr Crit Care Med. Author manuscript; available in PMC 2016 March 01. 

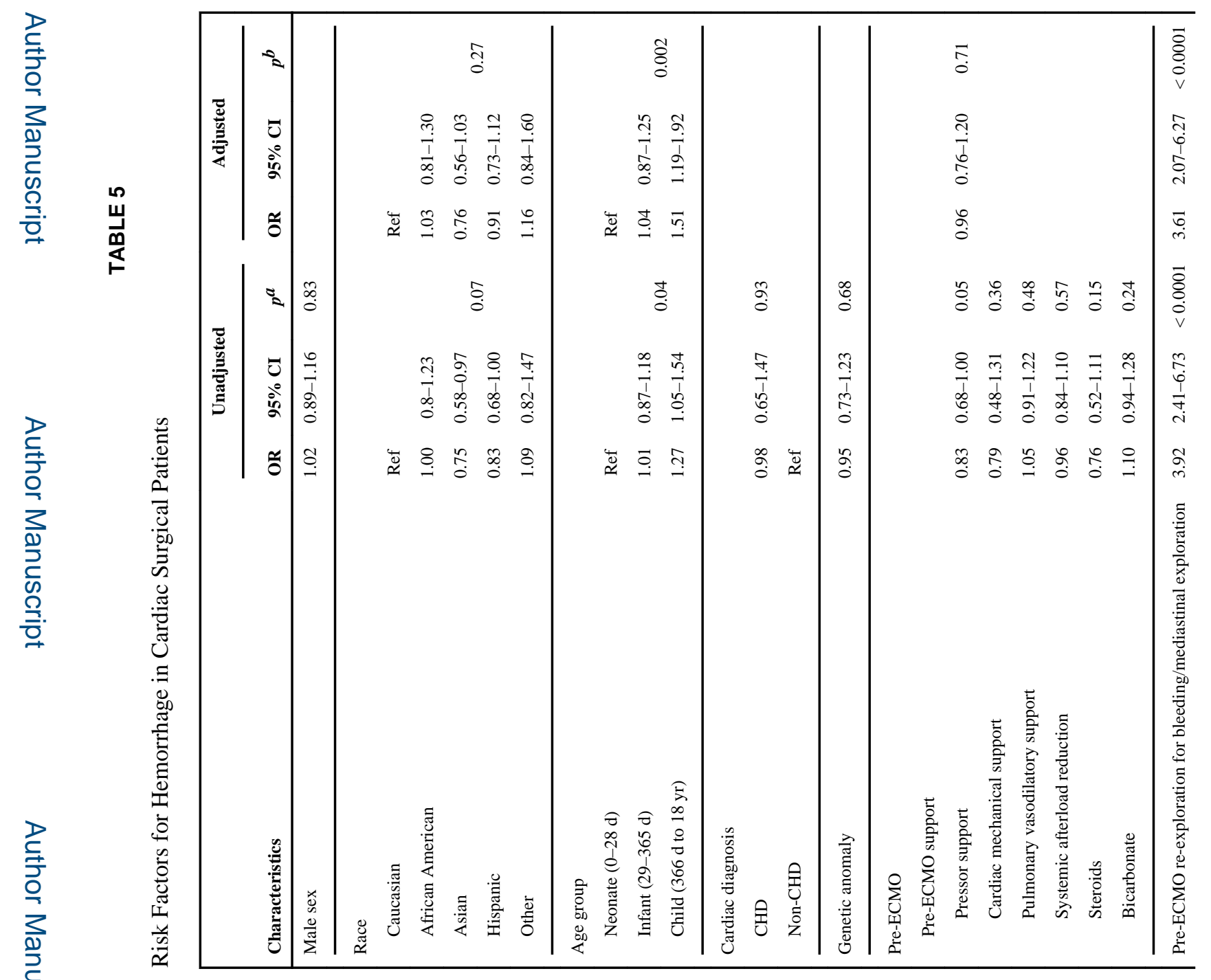

Pediatr Crit Care Med. Author manuscript; available in PMC 2016 March 01. 


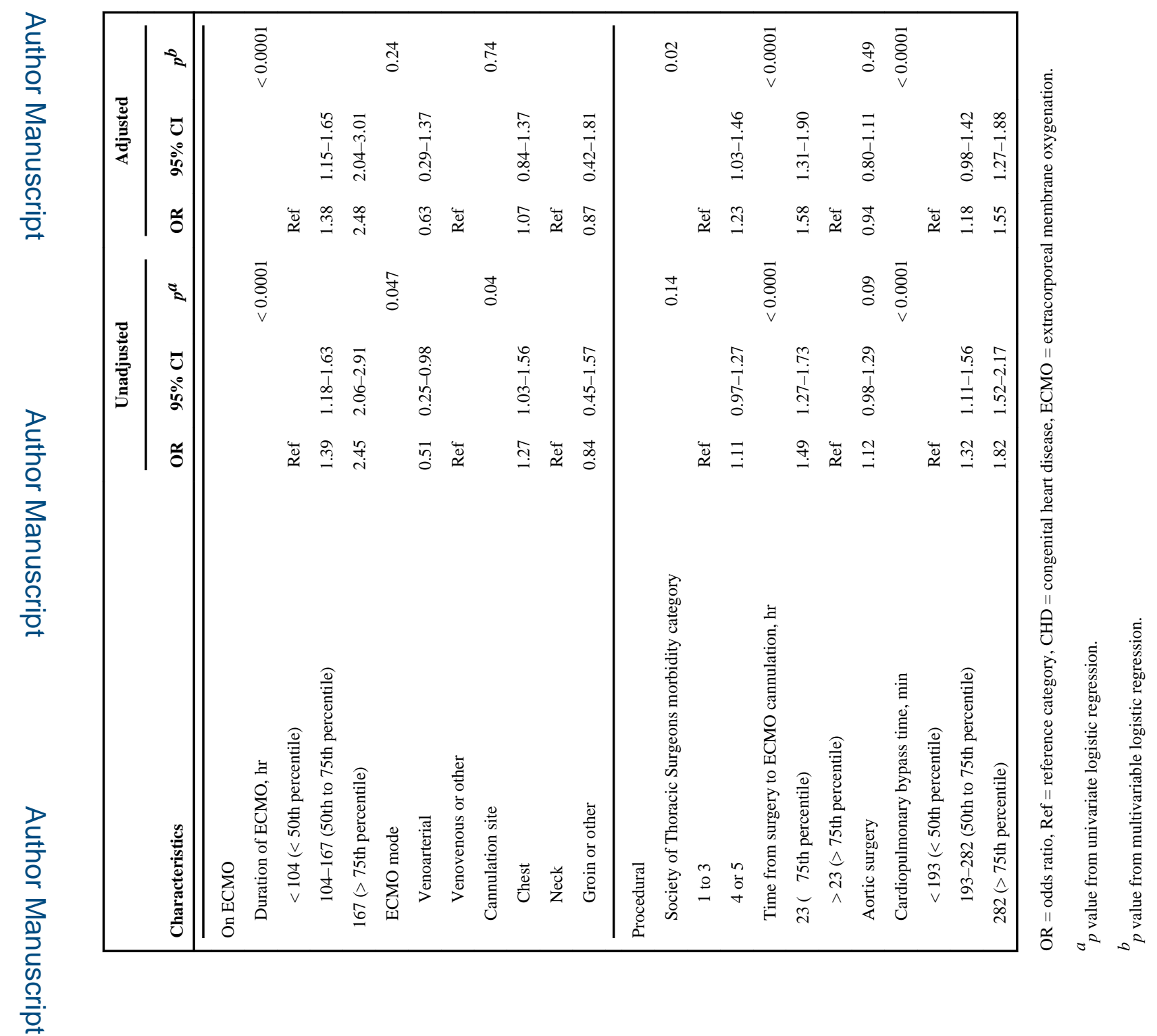

Pediatr Crit Care Med. Author manuscript; available in PMC 2016 March 01. 


\section{로을}

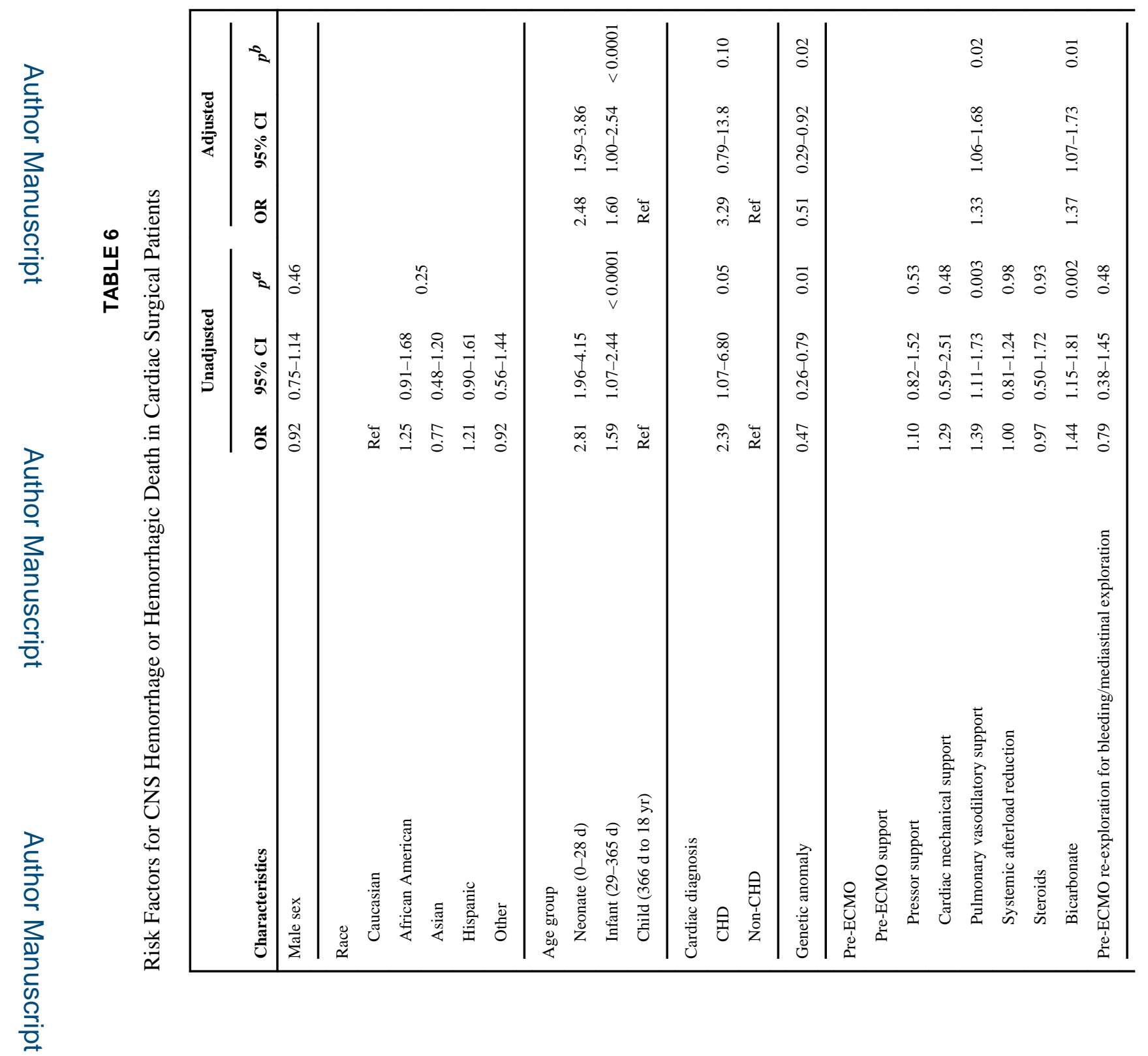

Pediatr Crit Care Med. Author manuscript; available in PMC 2016 March 01. 


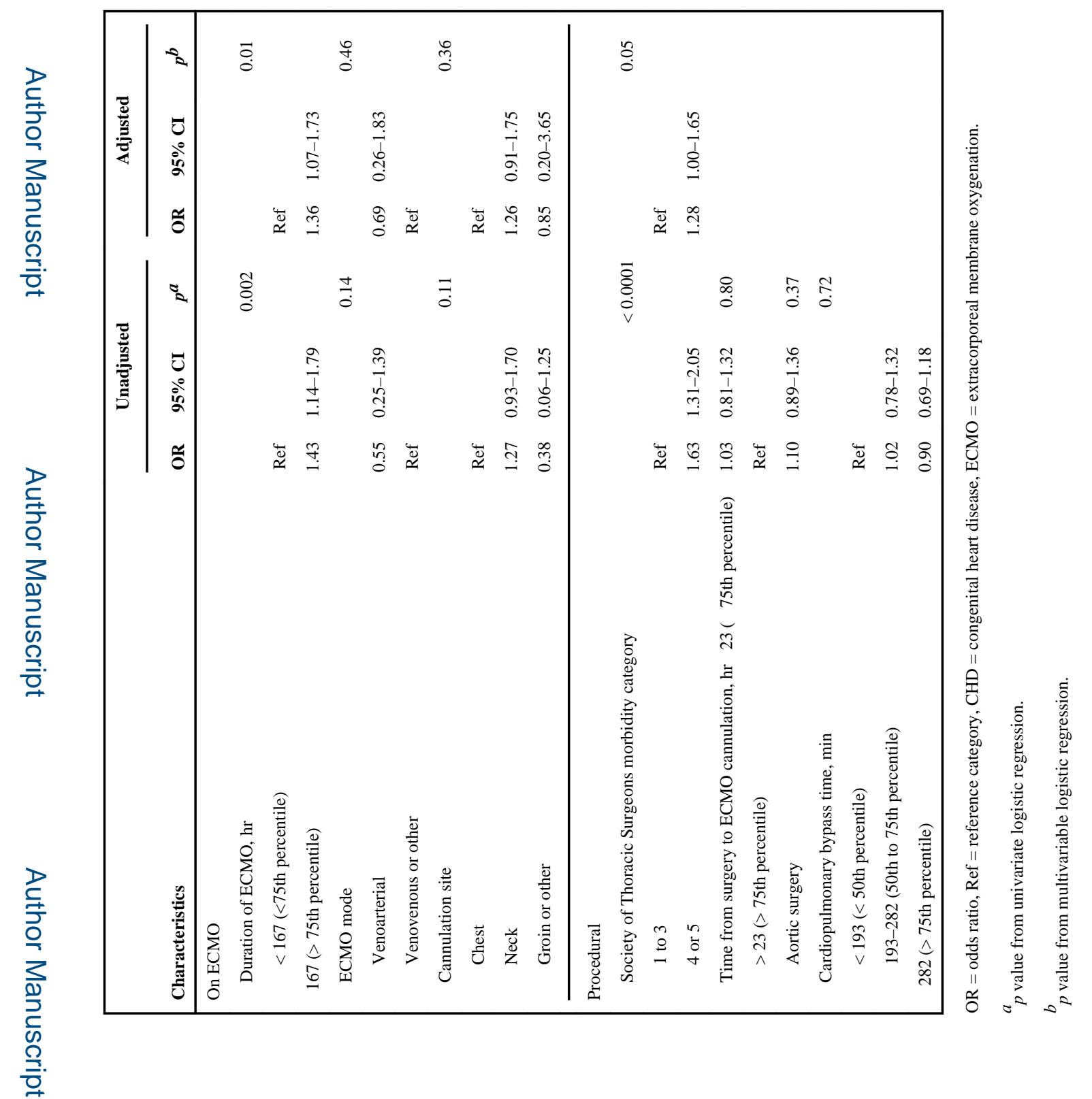

Pediatr Crit Care Med. Author manuscript; available in PMC 2016 March 01. 